\title{
Aprendizaje basado en juegos: incorporación de criterios sostenibles a través de la economía circular
}

Games based learning: incorporation of sustainable criteria through the circular economy

\author{
$\mathbf{M}^{\mathbf{a}}$ Esperanza Calvo Centeno \\ esperanza.calvo@universidadeuropea.es \\ Universidad Europea de Madrid. España \\ $\mathbf{M}^{\mathrm{a}}$ José Peset González iD \\ mariajose.peset@universidadeuropea.es \\ Universidad Europea de Madrid. España
}

\author{
Ma Pilar López Portillo \\ mpilar.lopez@universidadeuropea.es \\ Universidad Europea de Madrid. España \\ Eva Ropero Moriones \\ eva.ropero@universidadeuropea.es \\ Universidad Europea de Madrid. España
}

\section{Resumen}

En el presente trabajo se expone una actividad realizada a través de aprendizaje basado en juegos aplicado al conocimiento de la economía circular, que ha sido llevada a cabo con ocasión del ODS Day en la Universidad Europea de Madrid. El propósito de esta experiencia educativa se fundamenta en la necesidad de que la comunidad universitaria, y en especial los estudiantes, conozcan e interioricen modelos económicos sostenibles medioambientalmente. Se ha partido del modelo de economía circular, un nuevo paradigma de la gestión económica de los recursos, centrado en la reutilización de éstos y en la reducción de la generación de residuos. Para dar a conocer este modelo económico se ha desarrollado esta actividad de carácter transversal y metodología docente y tecnológica innovadoras. Para ello se ha aplicado el aprendizaje basado en juegos, así como un programa de realidad aumentada. La actividad se ha desarrollado a través de un juego en forma de yincana en la que los participantes se enfrentaron a retos en las distintas fases de la economía circular, contextualizada en el sector textil. Los resultados obtenidos muestran un interesante nivel de satisfacción de los participantes tanto con la metodología desarrollada como con el contenido de la actividad. Así mismo, se considera que el carácter transferible a otros centros docentes de esta iniciativa puede colaborar en promover de forma interactiva el conocimiento de la economía circular como modelo de sostenibilidad, así como a la sensibilización de los estudiantes frente a la responsabilidad medioambiental.

Palabras clave: economía verde; aprendizaje a través de la experiencia; educación para el desarrollo sostenible; tecnología educacional; investigación pedagógica.

\begin{abstract}
In the present work an activity applied to the learning of the circular economy through games-based learning is exposed, which has been carried out on the ODS day at Universidad Europea de Madrid. The purpose of this educational experience is based on the need of knowing and internalizing environmentally sustainable economic models for the university community, and especially the students. The activity is based on the circular economy model, a new paradigm of economic management of resources, focused on the reuse of these and on the reduction of waste generation. In order to publicize this economic model, this transversal activity with an innovative teaching and technological methodology has been developed. For this, game-based learning has been applied, as well as an augmented reality program. The activity was carried out through a game in the form of a gymkhana in which the participants faced challenges in the different phases of the circular economy, contextualized in the textile sector. The results obtained show an interesting level of satisfaction of the participants both with the methodology developed and with the content of the activity. Likewise, it is considered that the transferable nature of this initiative to other educational centers can help in promoting in an interactive way the knowledge of the circular economy as a model of sustainability, as well as the sensitization of students to environmental responsibility.

Keywords: green economics; experiential learning; education for sustainable development; educational technology; educational research.

Cómo referenciar este artículo / How to reference this article:

Calvo Centeno, M. E., López Portillo, M. P., Peset González, M. J., \& Ropero Moriones, E. (2020). Aprendizaje basado en juegos: incorporación de criterios sostenibles a través de la economía circular. Tendencias Pedagógicas, 36, pp. 176-186. doi: 10.15366/tp2020.36.14 


\section{Introducción}

La enseñanza actual se enfrenta a profundos cambios de muy diversa índole, tanto sociales como tecnológicos, que han convertido en obsoletas las estrategias docentes tradicionales en las que el docente se limitaba a impartir conocimiento, que era recibido de forma pasiva por el alumno. Las tecnologías de la información (TIC) y las constantes transformaciones sociales, han hecho indispensable la aplicación de nuevas estrategias, entre las que se encuentra el aprendizaje basado en juegos, que utiliza los mecanismos del juego con el objetivo de que los estudiantes adquieran conocimientos. La utilización de los juegos como enfoque pedagógico en el ámbito de la enseñanza se fundamenta en las teorías modernas de la educación que plantean que «el aprendizaje es más eficaz cuando es activo, experimental, situado, y basado en problemas» (García-Mundo, Vargas-Enríquez, Genero, \& Piattini, 2015, p. 73).

Actualmente, en el contexto educativo, las estrategias docentes basadas en los juegos hacen referencia a una tendencia sustentada en la unión del concepto de juego y aprendizaje (Marín, 2015). Así mismo, la integración de herramientas tecnológicas en interacción facilita la gestión del profesor para medir, seguir y controlar la estrategia de gamificación (Corchuelo-Rodríguez, 2018) y sirven para para motivar y potenciar el aprendizaje del alumno (Villalustre \& Del Moral, 2015).

Las experiencias fundamentadas en la aplicación de estrategias docentes basadas en juegos se han planteado con estudiantes de distintas etapas educativas, poniendo de manifiesto la eficacia de su implementación para la adquisición de conocimientos, tanto en educación superior (Bodnar, Anastasio, Enszer, \& Burkey, 2016; Borrego, Fernández, Blanes, \& Robles, 2017; Oliva, 2017) como en otras etapas no universitarias (Ke, 2014; De Soto, 2018; Del Moral, Guzmán Duque, \& Fernández García, 2018). En el ámbito de la enseñanza universitaria, el trabajo de Oliva (2017), pone de manifiesto que esta nueva herramienta educativa es un instrumento capaz de transformar la motivación extrínseca en intrínseca, logrando despertar la pasión del alumno por aprender.

En este sentido, las principales ventajas de la implementación de juegos en las actividades educativas son numerosas, entre las que cabe señalar, el aumento de la motivación de los estudiantes y su disposición para aprender (Bodnar et al., 2016; Borrego et al., 2017), la posibilidad de aprender en un entorno divertido y motivador (De Soto, 2018) que puede proporcionar al estudiante retroalimentación inmediata para promover su aprendizaje (Bodnar et al., 2016), así como la activación de la capacidad de reflexión (Ke, 2014). Por el contrario, se han detectado algunos inconvenientes como son la posibilidad de que el alumno centre su objetivo únicamente en ganar el juego, intentar hacer trampas, desmotivar a los alumnos «perdedores» o bien el hecho de que pueden consumir mucho tiempo, tanto en su realización como en la preparación de la actividad por parte del docente (Pisabarro y Vivaracho, 2018).

Siguiendo a Werbach y Hunter (2015), los juegos están estructurados por tres elementos: la dinámica, la mecánica y los componentes del juego. El primer elemento, la dinámica, se refiere a la visión general del juego. Comprende cuestiones como la denominada «narrativa del juego», que no es otra cosa que la historia en la que éste se fundamenta. También forman parte de la dinámica del juego las emociones que se espera provocar, como la curiosidad o la competitividad, así como las relaciones que desarrollará, es decir, las posibles interacciones con otros alumnos. Definida la visión global del juego, el siguiente elemento de éste a tener en cuenta es la mecánica, que comprende las reglas y su funcionamiento. A través de la mecánica del juego se delimitan elementos como son los desafíos que se plantean para superar un reto, si se juega individualmente o en grupo, el tipo de competición, posibles recompensas, retroalimentación de los logros obtenidos, y, por último, cómo se lleva a cabo la participación, si es secuencial, por azar, etc. Por último, el elemento final a definir está conformado por los componentes del juego que determinan los recursos con los que se lleva a cabo el mismo, dependiendo éstos del tipo de actividad de la que se trate (juego de ordenador, yincana, etc.). Los componentes son elementos como los logros a conseguir, las insignias que representen la consecución de dichos logros, la puntuación, límites temporales, etc.

Con base en todo lo anterior, un grupo de profesores de la Facultad de Ciencias Sociales y de la Comunicación de la Universidad Europea de Madrid ha considerado de interés implementar estrategias de aprendizaje basadas en juegos con el objetivo de incorporar criterios sostenibles sustentados en el modelo de economía circular. Este nuevo modelo económico supone un cambio de paradigma en la gestión de los recursos económicos frente al modelo tradicional lineal en el que el ciclo de vida 
de los productos está limitado a la secuencia extracción-producción-consumo-depósito de residuos. Como indica el Informe Cotec sobre economía circular en España:

\begin{abstract}
«el cambio se debe dar hacia sistemas que sean regenerativos a partir de su diseño, para mantener el valor de los recursos (materiales, agua, suelo y energía) y de los productos y limitando, exponencialmente, los insumos de materias primas y energía. Esto evitará la creación de residuos e impactos negativos derivados, mitigando las externalidades negativas para el medioambiente, el clima y la salud humana.» (Cotec, 2017, p. 22).
\end{abstract}

En este contexto, es importante resaltar que el modelo de economía circular se convierte en un elemento central en la consecución de varios de los Objetivos de Desarrollo Sostenible (ODS) de la Agenda 2030 (Naciones Unidas, 2015) entre los que cabe distinguir:

- Objetivo 8: Promover el crecimiento económico sostenido, inclusivo y sostenible.

- Objetivo 9: Construir infraestructuras resilientes, promover la industrialización inclusiva y sostenible y fomentar la innovación.

- Objetivo 12: Garantizar modalidades de consumo y producción sostenibles.

- Objetivo 13: Adoptar medidas urgentes para combatir el cambio climático y sus efectos.

- Objetivo 15: Promover el uso sostenible de los ecosistemas terrestres, luchar contra la desertificación, detener e invertir la degradación de los suelos y frenar la pérdida de la diversidad biológica.

El propósito de la actividad realizada es promover el desarrollo de estrategias pedagógicas basadas en juegos que sirvan para sensibilizar a la comunidad universitaria sobre cuestiones medioambientales relacionadas con el modelo de economía circular y su implementación en la vida real. El diseño de la actividad ha incorporado retos tecnológicos y se ha realizado coincidiendo con el ODS Day. La actividad tiene carácter grupal y se ha desarrollado a lo largo de las diferentes etapas de la economía circular aplicada al sector textil, planteando en cada una de ellas un reto que respondía a una pregunta sobre el conocimiento y el comportamiento de los alumnos ante la producción, el consumo y el reciclaje de su propia ropa.

\title{
2. Metodología
}

La actividad se desarrolló durante la semana de los ODS Days, celebrada en la Universidad Europea de Madrid en mayo de 2019, a lo largo de la cual se realizaron diversas acciones orientadas a implementar estrategias pedagógicas que permitieran a concienciar a la comunidad universitaria sobre la importancia de los Objetivos de Desarrollo Sostenible promovidos por Naciones Unidas. Dentro de este contexto, la actividad que se describe tuvo por finalidad plantear la sostenibilidad del actual sector textil y la divulgación de la economía circular como modelo alternativo sostenible.

Se ha partido de que la sostenibilidad es un objetivo para implementar en toda la comunidad educativa, por lo que esta actividad se ha dirigido a todos sus componentes, tanto estudiantes, como docentes y empleados no docentes. Tuvo lugar en el Campus de Villaviciosa de Odón (Madrid), en donde se ubicaban Facultades y Escuelas de diferentes ámbitos educativos (Ciencias Sociales y de la Comunicación, Ingenierías, Medicina, Grados Superiores de Formación Profesional, etc.), lo cual posibilitó una heterogeneidad de participantes que resultó muy enriquecedora.

Esta actividad se realizó en un espacio común al aire libre a lo largo de la mañana del mencionado ODS Day, lo que permitió dar una mayor visibilidad a la actividad y, además, animó a numerosos participantes a concurrir a la misma.

Por último, al tratarse de una actividad voluntaria, fue percibida de forma más lúdica y menos reglada, lo que supuso otro elemento que estimuló la participación.

El formato elegido para llevar a cabo la actividad fue una yincana, en la que se plantearon una serie de pruebas que se realizaron por equipos. Dichas pruebas implicaban retos que debían ser superados por los participantes y al finalizar la misma los ganadores obtuvieron un premio.

Los equipos estaban formados por un número variable entre 3 y 5 componentes. Tras su inscripción como grupo, con los datos de sus miembros, se les entregaban las instrucciones para descargarse la aplicación de realidad aumentada Zappar (para la elaboración del contenido se utiliza la herramienta 
Zapworks). Los alumnos se tenían que instalar la aplicación en el móvil, que debían utilizar desde su móvil, y que les permitía acceder al material de las pruebas.

Estas pruebas estaban fundamentadas en la estructura de la economía circular y las diferentes etapas en las que ésta se desarrolla, aplicado a la industria textil: los materiales textiles, la fabricación de ropa, su distribución, el consumo responsable, el reciclaje y tratamiento de residuos textiles.

De esta forma, se dispusieron seis espacios, uno por cada etapa. En cada una de ellas los participantes debían superar un reto concreto, relacionado con el tema de cada una de las fases de la economía circular. La resolución de cada reto fue evaluada e incorporada a un «pasaporte» en el que se sumaban los resultados obtenidos a lo largo del recorrido de la yincana.

Una vez realizadas las actividades de todas las etapas, los grupos debían dirigirse a la mesa de finalización donde quedaba registrado, junto con sus datos, el resultado total de todas las pruebas. Acabada la actividad, se analizaron los resultados para identificar los tres grupos con mayor puntuación, que obtuvieron un premio. Los participantes conocían desde el principio la existencia de estos premios, lo que supuso un aliciente para una mejor resolución de los retos.

Todas las actividades de la yincana se vincularon a través de la aplicación de realidad aumentada Zappar, en la que aparecía información extra sobre una imagen real. Esta información se visualizaba tras enfocar el móvil a unos carteles informativos de cada etapa, utilizando la mencionada app. La información podía ser un audio, un vídeo o una encuesta online, según la etapa que correspondiera y los participantes necesitaban acceder a ella para poder llevar a cabo cada uno de los retos propuestos.

Para estructurar las diferentes etapas de la yincana se siguió la secuencia que se muestra en el siguiente esquema:

- Etapa de la economía circular > Objetivo: pregunta general que deben responder los participantes en relación con esta etapa > Información sobre el objetivo > Reto a resolver > Evaluación de la resolución del reto.

Siguiendo esta secuencia, la organización de los distintos espacios de las seis etapas definidas fue la siguiente.

\subsection{Primera Etapa: Diseño ¿De qué materiales está hecha tu ropa?}

La economía circular tiene como objetivo mantener durante el mayor tiempo posible el valor de los productos, los materiales y los recursos, reduciendo al mínimo la generación de residuos. El ciclo de vida de un producto comienza con su diseño, y dentro de esta etapa, los materiales que lo componen son esenciales para obtener un producto sostenible. Dentro de la economía circular, el ecodiseño tiene en cuenta no solo el uso que el consumidor va a dar al bien, sino también el impacto medioambiental de la obtención de sus componentes, así como que dicho impacto sea mínimo cuando la vida útil del bien finalice, permitiendo su reciclaje o reutilización.

Por tanto, el objetivo de esta etapa era que los participantes tomaran conciencia de la importancia de los materiales con los que se fabrica la ropa. Para ello, los alumnos disponían de un video de elaboración propia al que accedían a través de la aplicación de la realidad aumentada, en el que se explicaba el origen de los principales tipos de materiales textiles, incidiendo en la huella medioambiental a que da lugar su producción y desecho.

El reto consistía en que cada componente del grupo debía buscar en la etiqueta de una de las prendas que vestía, los materiales de esta. Las prendas se valoraron en base al porcentaje de cada material que contenían, así como en función de la sostenibilidad de cada uno de ellos. Fueron menos valorados los materiales considerados menos sostenibles (sintéticos y artificiales) y mejor puntuados los más sostenibles (naturales, reciclados y ecológicos). Finalmente, se calculaba la media de la puntuación obtenida por las prendas de todos los participantes del grupo, que se anotaba en el «pasaporte» del mismo.

\subsection{Segunda Etapa: Producción ¿En qué condiciones se fabrica tu ropa?}

En el modelo de economía circular, la etapa de producción incide no solo en la sostenibilidad medioambiental de la misma (uso de materiales reciclados o sostenibles, energías renovables, incremento de la vida útil de los productos, etc.), sino que para desarrollar todo su potencial necesita de un 
entorno social sostenible y justo. En el caso de la industria textil, la situación es especialmente preocupante puesto que de los aproximadamente 60 millones de personas que trabajan en la misma, más del 50\% no alcanza el salario mínimo (Ethics, 2017). A ello hay que añadir condiciones laborales precarias, con largas jornadas laborales y reducida o nula protección de los derechos de los trabajadores, en especial mujeres y niños.

Por tanto, el objetivo de esta etapa era hacer reflexionar a los participantes de las condiciones laborales de los países en los que se fabricaba su ropa y concienciarlos de la necesidad de alcanzar unas condiciones justas en los mismos.

Para ello se les proporcionó información a través de realidad aumentada disponible en la web de la organización Ropa Limpia sobre elementos mínimos que debe comprender un salario digno y video muy sintético sobre las condiciones laborales de países subdesarrollados en los que mayoritariamente se fabrica la ropa que se consume a nivel mundial.

El reto consistió en que los participantes conocieran el grado de cumplimiento de los derechos laborales del país en el que se había fabricado la prenda que habían seleccionado en la etapa anterior. Para ello se instaló un mural con un mapamundi elaborado por la ITUC (International Trade Union Confederation) y en el que se clasificaba a los países en función del cumplimiento de los derechos laborales. Los participantes debían buscar el país de origen de su prenda y situarlo en el mapa con un alfiler. De esta forma pudieron visualizar gráficamente los países que mayoritariamente fabricaban la ropa habitualmente utilizada.

La evaluación del reto se llevó a cabo a través del mencionado ranking, de forma que las prendas fabricadas en países con un mejor cumplimiento de derechos obtuvieron una mayor puntuación.

\subsection{Tercera Etapa: Distribución ¿Dónde se fabrica tu ropa?}

Según la Agencia Europea del Medioambiente (AEMA, 2016) el transporte es responsable de más de una cuarta parte de las emisiones totales de gases de efecto invernadero de la UE, de los que el $70 \%$ provienen del transporte por carretera y el resto es producido por del transporte marítimo y aéreo. Además, mientras que la industria y la producción de energía han reducido sus emisiones desde 1990, sin embargo, las emisiones del sector del transporte se han incrementado.

En la economía circular la distribución de los productos es un elemento significativo del proceso económico, debido a su implicación en la sostenibilidad medioambiental del mismo. Por ello, una industria textil sostenible implica un consumo de moda cuya huella de carbono sea reducida. Sin embargo, la globalización ha provocado que determinados países se especialicen en la producción textil, de modo que sea necesario el traslado desde estos lugares a los puntos de consumo, incrementando el transporte y, por tanto, las emisiones de gases de efecto invernadero.

Por tanto, en esta etapa se trataba de que los participantes tomaran conciencia de la huella de carbono que implica el consumo de determinadas prendas y su impacto en el medioambiente.

Inicialmente, accedían a un vídeo de elaboración propia a través de la aplicación de realidad aumentada, en el que se les explicaba la relación entre globalización, transporte y emisiones de $\mathrm{CO}_{2}$. El reto consistía en que comprobaran donde se había fabricado la prenda de ropa que cada uno de ellos había elegido en las anteriores etapas. A través de una calculadora de kilómetros que se les proporcionaba, debían obtener la distancia que había tenido que recorrer esa prenda hasta llegar a un puerto español (se eligió el de Valencia por ser uno de los de mayor tránsito de la península).

La evaluación del reto se realizó en función de la distancia que había tenido que recorrer la prenda hasta el punto en el que el participante la había comprado, de forma que cuanto menor fuera ésta, más sostenible sería la prenda y mejor la puntuación.

\subsection{Cuarta Etapa: Consumo Responsable ¿Qué tipo de consumidor eres?}

Durante las últimas décadas el modelo imperante desarrollado en la industria textil se ha orientado a fomentar el consumo rápido y constante de productos, como sistema para maximizar la satisfacción del cliente. En la economía circular, el consumo responsable es un elemento sustancial del proceso que implica la misma. En oposición a un consumo basado en la dualidad Usar-Tirar, la economía circular plantea un cambio en los hábitos de consumo de forma que éstos se ajusten a las necesidades reales de los consumidores y que seleccionen alternativas respetuosas con el medio ambiente. 
Por tanto, el objetivo de esta etapa era conseguir que los participantes fueran conscientes de sus hábitos de consumo de ropa y el impacto medioambiental de éstos. Para ello, se propuso a los componentes de los grupos una reflexión sobre sus costumbres y rutinas de compra de ropa a través de un cuestionario elaborado con la herramienta Playbuzz y al que accedían a través de la aplicación de realidad aumentada. En el mismo se les planteaban cuestiones como cuantas prendas se compraban mensualmente, si consideraban esas adquisiciones necesarias, si tenían en cuenta el impacto medioambiental de las prendas adquiridas o las condiciones laborales del país en el que se habían fabricado, si los materiales de los que estaba compuesta eran sostenibles, etc. En función de las respuestas se elaboraron cuatro perfiles de consumidores basados en criterios sostenibles y de responsabilidad en el consumo, con los que se evaluaron a los participantes.

\subsection{Quinta Etapa: Reutilización de ropa ¿Qué haces con tu ropa cuando no la quieres?}

En el proceso de la economía circular, el tratamiento y el reciclaje de residuos forma parte de los elementos básicos que sustentan su concepción. En el contexto de la industria textil, cuando el consumidor quiere desechar una prenda las alternativas de reutilización de la ropa son variadas. La ropa puede volver al circuito de uso de esta, a través de mercados de segunda mano o donaciones a entidades específicas (ONGs, Iglesias, etc.), o bien puede ser rediseñada para poder seguir siendo usada. También puede trasladarse para ser reciclada y utilizada como materia prima de otros productos. En cualquier caso, la peor alternativa siempre será enviarla a la basura convencional, cuyo coste de incineración e impacto medioambiental es muy alto.

El objetivo de esta etapa era mostrar a los participantes las diferentes alternativas de reutilización de la ropa que permiten incrementar la vida de ésta. Para ello, se les mostraba a través de realidad aumentada una infografía de elaboración propia que sintetizaba las opciones posibles para dar salida a la ropa una vez ya no se va a utilizar.

El reto consistía en realizar un cuestionario elaborado a través de la aplicación Play-buzz en el que los participantes mostraban su conducta cuando decidían deshacerse de su ropa. En función de las respuestas se elaboraron distintos perfiles y se evaluó su comportamiento, siendo el más positivo el que presentaba una actuación que incrementaba la vida de las prendas y, por tanto, se consideraba más sostenible.

\subsection{Sexta Etapa: Reciclaje de Residuos ¿Qué ocurre con tu ropa cuando acaba en la basura?}

La última fase que cierra el círculo de la vida de un producto en la economía circular es el reciclaje de los residuos, cuyo fin es reducir la utilización de nuevos recursos en los procesos productivos. Según la Comisión Europea (citado en Amigos de la Tierra, 2015), los europeos desechan 5,8 millones de toneladas de ropa anuales de los que el $75 \%$ acaba en vertederos, lo que implica que solo se recicla un reducido porcentaje de la ropa desechada. El caso español es más llamativo, puesto que según Asirtex (2016) cada año se desecha aproximadamente $10 \mathrm{~kg}$ de ropa usada por persona, de los que solo uno se recicla.

El objetivo de esta etapa era sensibilizar a los participantes de la necesidad de una adecuada separación de residuos, para lo cual se les mostraba a través de realidad aumentada un video de producción propia sobre la descomposición de la ropa, en donde se explicaban las diferentes alternativas cuando se decide desechar la misma (punto limpio, contenedor de ropa a pie de calle o basura) y los beneficios o perjuicios que cada una de ellas, haciendo hincapié en la duración de tiempo de descomposición de los diferentes tipos de materiales, en caso de que se optara por no reciclar.

El reto consistía en que los participantes revisaran los materiales de su prenda y calcularan el tiempo de descomposición de esta. Cuanto más largo fuera dicho periodo, peor fue la evaluación obtenida.

Tras acabar esta última etapa de la yincana, los participantes se dirigían a la mesa de inscripción para entregar su pasaporte, en donde figuraba la puntuación obtenida en cada una de ellas. De esta forma, se calculaba el total de la puntuación, que quedaba registrada y permitía la elección de los equipos ganadores una vez finalizada la actividad. Así mismo, se les entregaba un documento en el que se proponía seis recomendaciones para vestir de forma sostenible basadas en las etapas de la yincana: consumir ropa fabricada con materiales sostenibles, procedente de lugares donde se respeten 
los derechos de los trabajadores, prendas que dejen la menor huella de carbono posible, plantearse un consumo responsable y reciclar adecuadamente la ropa cuando ya no se vaya a utilizar.

\section{Resultados}

El propósito de la actividad realizada es promover el desarrollo de estrategias pedagógicas basadas en juegos que sirvan para sensibilizar a la comunidad universitaria sobre cuestiones medioambientales relacionadas con el modelo de economía circular y su implementación en la vida real. Con base en lo anterior, para poder determinar si las estrategias pedagógicas implementadas han servido para propiciar un acercamiento al concepto de economía circular se han establecido los siguientes resultados de aprendizaje:

- Llevar a cabo una primera aproximación práctica en el ámbito universitario que muestre en qué consiste la economía circular.

- Comprender, a través de un medio tan próximo como la propia ropa, cuáles pueden ser las consecuencias sobre el medioambiente y la sostenibilidad de determinadas decisiones individuales.

- Desarrollar la conciencia de los alumnos acerca de la utilidad de las herramientas tecnológicas y nuevas realidades (aumentada, virtual) para incentivar y generar conocimiento a largo plazo.

Al finalizar la actividad se propuso a los participantes un cuestionario sobre la actividad con el objeto de conocer el grado de satisfacción de estos con la actividad y la consecución de los objetivos propuestos. Además, se les proporcionó la opción de comentarios de forma abierta.

La encuesta se realizó en una escala Likert, con 5 niveles respuesta. La encuesta se realizaba también a través de la aplicación de realidad aumentada, y su respuesta era voluntaria. A pesar de su voluntariedad, la encuesta fue contestada por 60 participantes.

Preguntados en primer lugar acerca de su grado de satisfacción con la actividad, se observa que un $93,3 \%$ de los encuestados se mostró satisfecho o muy satisfecho con la actividad, siendo la moda la respuesta que mostraba más alta satisfacción, con un $68,3 \%$.

Figura 1.

Resultados del grado de satisfacción con la actividad

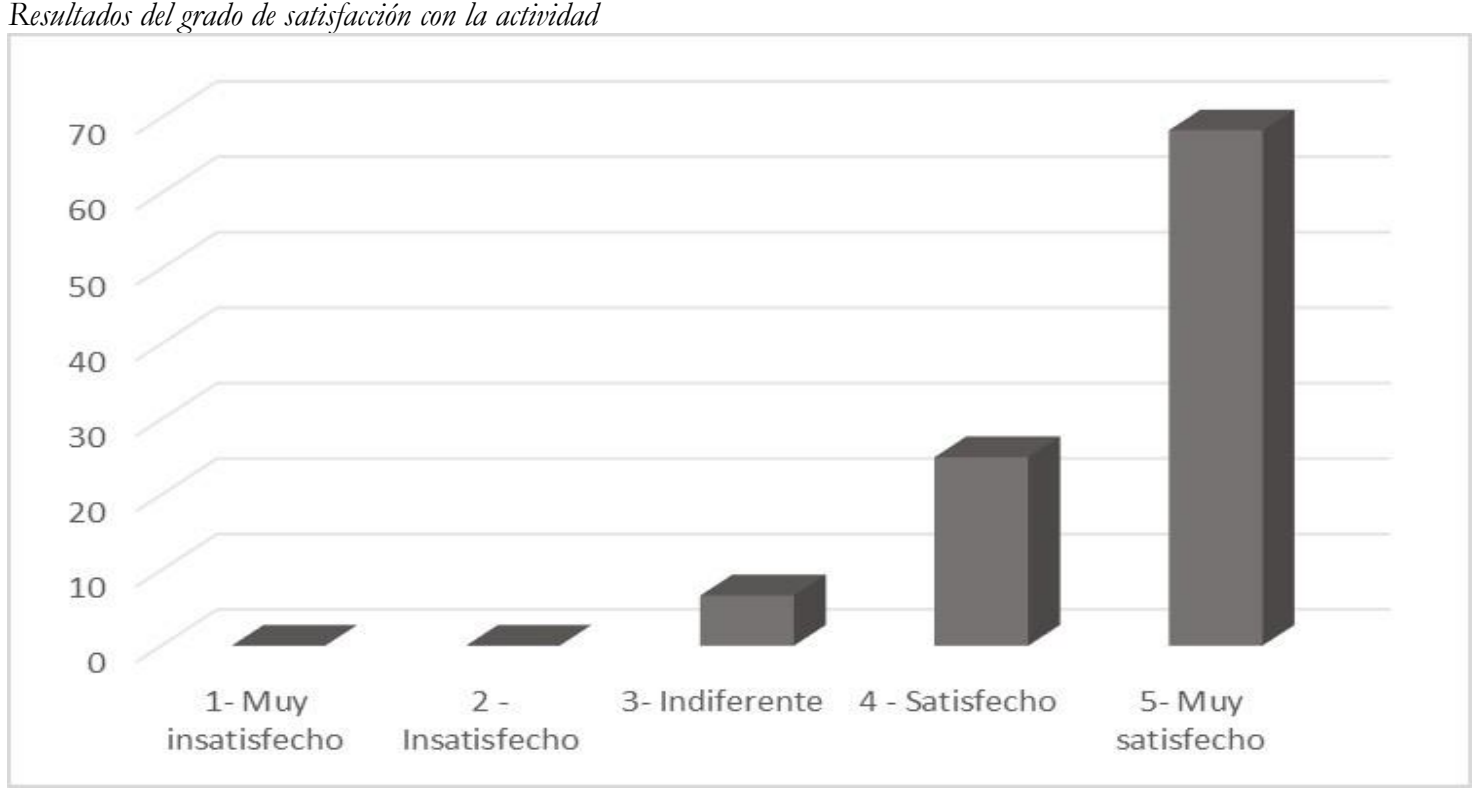

Fuente: Elaboración propia.

Posteriormente se les consultó acerca de la utilidad de la metodología con respecto a la actividad que se había desarrollado, para lo cual se realizaron dos cuestiones, sobre cómo de adecuada había sido la metodología para los contenidos que se habían mostrado y cómo de útil para comprenderlos mejor. Estos resultados se encuentran recogidos en la Figura 2, donde se observa que la mayor 
frecuencia demuestra que los encuestados se mostraron muy de acuerdo con el uso de la metodología utilizada en la actividad. En total el porcentaje de personas que estaban de acuerdo o muy de acuerdo tanto con que "la metodología es adecuada para los contenidos" como con "la actividad me ha resultado útil para comprender mejor los contenidos" era igual a $88,3 \%$, con un encuestado más que se mostró muy de acuerdo con la primera expresión.

Los participantes que se mostraron en desacuerdo con lo adecuada de la actividad representaban un $3,3 \%$.

Figura 2.

Resultados obtenidos sobre la utilidad de la actividad en cuanto a metodología y comprensión de los contenidos

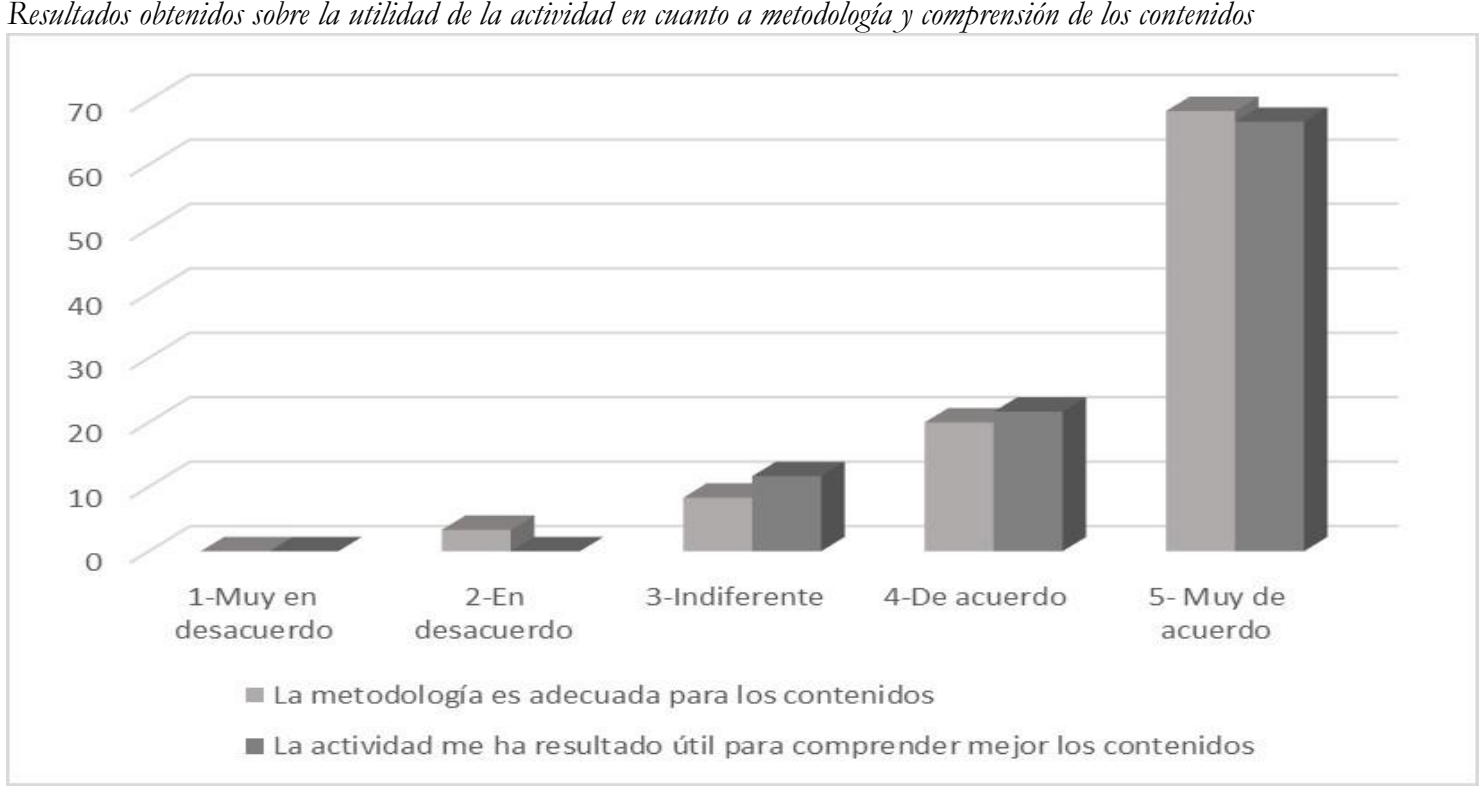

Fuente: Elaboración Propia.

Ante la cuestión de si el uso de nuevas tecnologías aumenta la motivación, los resultados se presentan en la siguiente gráfica:

Figura 3.

Resultados sobre la idea de motivación que el tipo de actividad y los recursos tecnológicos utilizados ba despertado a los alumnos

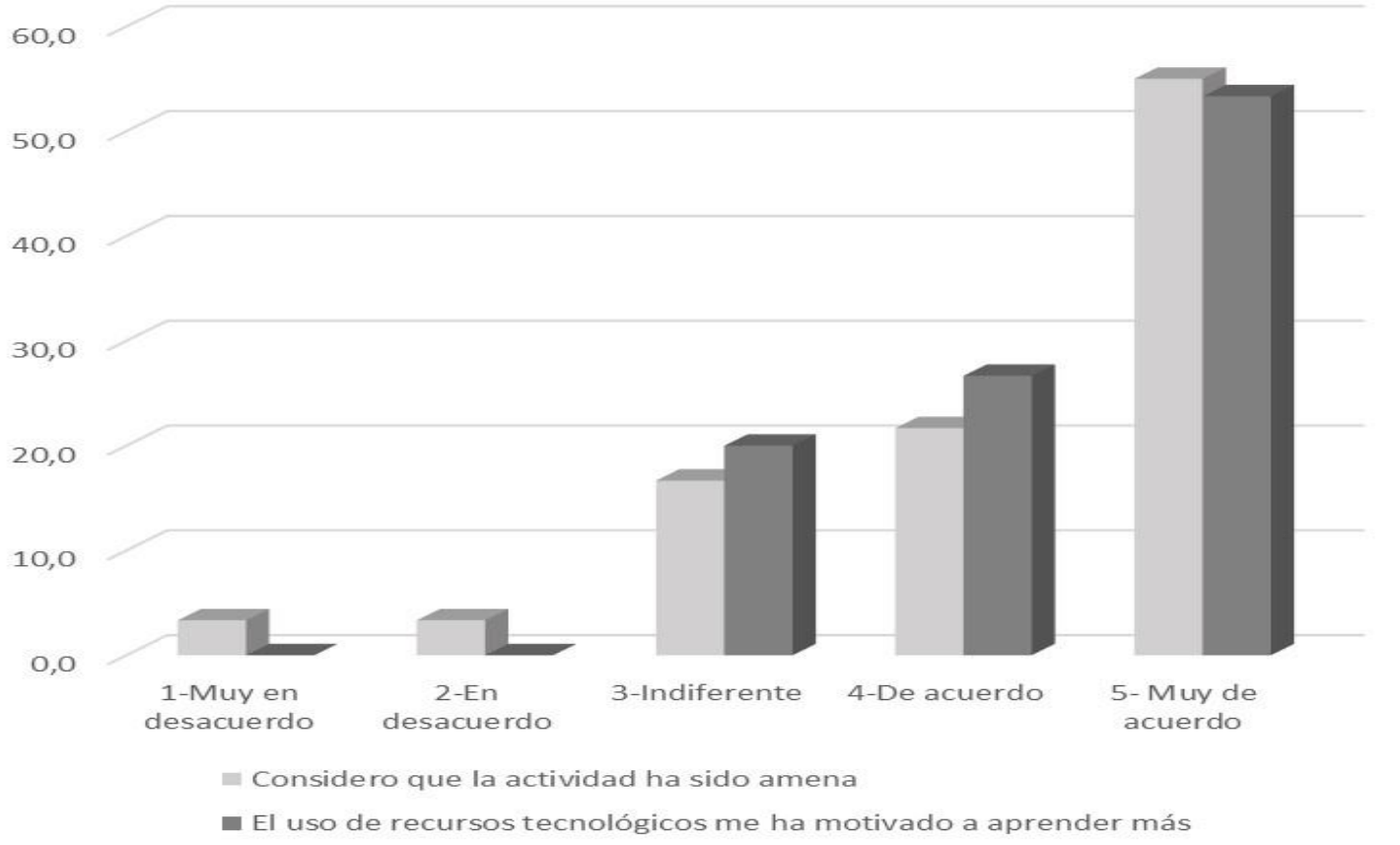

Fuente: Elaboración Propia. 
El porcentaje de participantes que estaban de acuerdo o muy de acuerdo con «la actividad ha sido amena» fueron $76,7 \%$, mientras que con «el uso de recursos tecnológicos me ha motivado a aprender más» fueron $80 \%$. Una vez más, una mayoría estaba «muy de acuerdo» con las afirmaciones, de lo que podemos deducir que una mayoría de participantes aumentó su motivación gracias al uso de tecnologías innovadoras.

Un porcentaje minoritario $(6,6 \%)$ contestó que la actividad no le había resultado amena, a pesar de que nadie mostró desacuerdo con la afirmación acerca de la motivación para aprender.

En el apartado de respuesta abierta se recibieron comentarios acerca de diversas opiniones sobre diferentes aspectos de la actividad. De entre todos los comentarios recibidos, podemos destacar los siguientes aspectos:

- La actividad resultó, en general, gratificante y motivadora.

- La actividad presentaba un enfoque diferente a otro tipo de actividades y esto les pareció más motivador para aprender.

- Gracias a la actividad comprendieron la importancia y utilidad de cuidar el medio ambiente en su vida diaria.

- Descubrieron la importancia de la economía circular y su relación con las decisiones que tomamos en la vida diaria.

- La actividad consiguió activar o aumentar su conciencia medioambiental.

- Destacaron la importancia de realizar la actividad mediante un juego en el que se aunaba tecnología y práctica real junto con la posibilidad de obtener premios en la profundidad con la que adquirieron el aprendizaje.

Los resultados obtenidos permiten concluir que se han alcanzado los resultados de aprendizaje previamente establecidos. La estrategia pedagógica implementada para realizar la actividad ha mostrado ser útil, tanto para interiorizar los conocimientos adquiridos sobre la economía circular, como para concienciar sobre los efectos que pueden tener las decisiones personales sobre las cuestiones sociales, medioambientales y económicas que conforman la sostenibilidad.

\section{Conclusiones}

El interés de este trabajo se fundamenta en la incorporación de criterios sostenibles, sustentados en la economía circular, utilizando como estrategia pedagógica el aprendizaje basado en juegos a través de la realidad aumentada. Dicho interés nace de la consideración del agotamiento del modelo tradicional de producción y consumo, basado en la concepción de que los recursos son inagotables y los desechos que genera el consumo son infinitamente asumibles por el planeta. En este sentido, la economía circular se presenta como un nuevo paradigma que abarca todo el proceso productivo, de consumo y de retorno de los recursos para su reutilización, posibilitando una alternativa al agotamiento de los inputs productivos y a las externalidades negativas que se generan en el ámbito medioambiental y social. Además, como se ha puesto de manifiesto a lo largo de este trabajo, esta nueva concepción de la producción y consumo es una pieza fundamental para lograr alcanzar los ODS de la Agenda 2030 de la ONU relacionados con la sostenibilidad medioambiental y sus implicaciones en las cuestiones sociales y económicas.

Para llevar a cabo la implementación de esta experiencia docente se ha buscado una metodología basada en juegos por resultar motivadora, activa y en sintonía con las TIC. Se ha estimado que el uso del aprendizaje basado en juegos resulta una metodología que cubre los objetivos docentes propuestos y que, a través de retos y desafíos, permite alcanzar objetivos de aprendizaje y desarrollar competencias transversales de forma motivadora y proactiva, así como conseguir que se mantengan en el tiempo.

Al realizarse la actividad sobre el sector textil, se han podido trabajar conceptos relacionados con diferentes estrategias de reutilización. Además, los participantes podían visualizar fácilmente, cuál era su contribución a la sostenibilidad, al revisar qué tipo de ropa se consume y como se desecha, así como replantearse su compromiso hacia el futuro.

Las principales conclusiones obtenidas a través de la actividad desarrollada se sintetizan en las siguientes: 
- Se ha proporcionado a los participantes una visión global, y a la vez muy personal, del concepto de economía circular, al abarcar las diferentes etapas de esta dentro de un todo y no como elementos individuales, incidiendo en la idea de "circularidad" del modelo en el cual todas las etapas son necesarias e importantes para que éste funcione.

- La organización de las etapas, siguiendo el esquema de objetivo de aprendizaje > información $>$ reto $>$ resolución $>$ evaluación $>$ recompensa, permitió que los participantes aprendieran de forma más motivada, al introducir elementos clásicos de los juegos como son los desafíos y la recompensa.

- El hecho de necesitar llevar a cabo la actividad con herramientas tecnológicas y nuevas realidades, como la aumentada y la virtual, les proporcionó no solo conocimiento de estas, sino una conciencia práctica de su utilidad y el desarrollo de competencias tecnológicas.

- El planteamiento de las actividades con un enfoque muy personal ha proporcionado una percepción de gran proximidad al conocimiento adquirido, es decir, el "saber" ha dejado de ser algo externo al alumno, convirtiéndose en una cuestión cercana y, por tanto, más interesante. Esta proximidad a la problemática planteada ha permitido un aumento de la conciencia sobre los problemas medioambientales y la sostenibilidad de los participantes.

- Todas estas características de la actividad (uso de juegos, nuevas tecnologías, proximidad) hacen que el aprendizaje obtenido por los participantes active la concienciación.

Son varias las cuestiones que se pueden mencionar respecto de las limitaciones de esta experiencia docente.

En primer lugar, es interesante destacar que se trata de una actividad docente cuyo objetivo es la sensibilización de cuestiones que están directamente vinculadas con cambios culturales y sociales y, que, en ese sentido, con esta actividad se pretende una primera toma de contacto con los mismos. Por tanto, se hace necesaria la continuidad de actividades con similares objetivos, tanto a nivel curricular como transversal, así como el seguimiento de sus resultados, para alcanzar dichos objetivos con toda la profundidad que esta temática requiere.

En segundo lugar, se ha de comentar que solo se han incorporado datos pertenecientes al año 2019, al ser el primer año en el que se ha realizado la actividad referida al sector textil. Futuras ediciones de la actividad permitirán alcanzar resultados más concluyentes y un seguimiento de los posibles cambios de comportamiento por parte de los participantes. Por último, se ha de señalar que esta actividad se ha circunscrito únicamente al ámbito educativo de la Universidad Europea de Madrid. No obstante, se considera que es importante resaltar que, como indica Arquero (2012), en la investigación sobre experiencias docentes su relevancia está directamente relacionada con lo que denomina transferibilidad de dichas experiencias, es decir, en que éstas puedan ser llevadas a cabo en otras universidades o entornos educativos. En este sentido, se considera clara la relevancia de esta experiencia ya que por su formato y diseño puede realizarse en diversos ámbitos docentes, alcanzando los objetivos propuestos. De hecho, esta actividad puede presentar nuevas versiones, aplicándose a sectores o industrias concretos de la economía especialmente agresivos medioambientalmente o contaminantes, y que proporcionen una visión de la economía circular como eje central de la sostenibilidad.

\section{Referencias}

Agencia Europea del Medio Ambiente (2016). Hacia una movilidad limpia e inteligente. Transporte y medio ambiente en Europa. Copenhague: AEMA. Recuperado de https://www.eea.europa.eu/es/publications/senales-2016-informe-completo

Amigos de la Tierra (2015). Menos es más. Eficiencia de los recursos mediante la recogida, reutilización y reciclaje de residuos de aluminio, algodón y litio en Europa. Vienna: Global 2000 Verlagsges. Recuperado de https://www.tierra.org/wp-content/uploads/2015/03/menos_es_mas.pdf

Arquero, J. L. (2012). Investigación en docencia de la contabilidad: consideraciones y consejos. Revista AECA, 99, pp. 4-7.

Asociación Ibérica de Reciclaje Textil (2016). La ropa usada: tendencias de un mercado emergente. Valencia: ASIRTEX. Recuperado de https://www.asirtex.org/wp-content/uploads/2019/05/La-RopaUsada.-Tendencias-de-un-mercado-emergente.-El-residuo-olvidado-en-el-siglo-XXI..pdf 
Bodnar, C. A., Anastasio, D., Enszer, J. A., \& Burkey, D. D. (2016). Engineers at play: Games as teaching tools for undergraduate engineering students. Journal of Engineering Education, 105(1), pp. 147-200. doi: 10.1002/jee.20106

Borrego, C., Fernández, C., Blanes, I., \& Robles, S. (2017). Room escape at class: Escape games activities to facilitate the motivation and learning in computer science. JOTSE: Journal of Technology and Science Education, 7(2), pp. 162-171. doi: 10.3926/jotse.247

Comisión Europea (2015). Comunicación de la Comisión al Parlamento Europeo, al Consejo, al Comité Económico y Social Europeo y al Comité de las Regiones. 'Cerrar el círculo: un plan de acción de la UE para la economia circular'. Bruselas: COM (2015) 614 final.

Corchuelo-Rodríguez, C. A. (2018). Gamificación en educación superior: experiencia innovadora para motivar estudiantes y dinamizar contenidos en el aula. Edutec. Revista Electrónica de Tecnología Educativa, 63, pp. 29-41. doi: 10.21556/edutec.2018.63.927

COTEC (2017). Situación y evolución de la economía circular en España. Madrid: Fundación Cotec para la Innovación.

De Soto García, I. (2018). Herramientas de gamificación para el aprendizaje de ciencias de la tierra. EDUTEC: Revista Electrónica de Tecnologia Educativa, 65, pp. 29-39. doi: 10.21556/edutec.2018.65.1143

Del Moral, M., Guzmán Duque, A., \& Fernández García, L. (2018). Game-Based Learning: Increasing the Logical-Mathematical, Naturalistic, and Linguistic Learning Levels of Primary School Students. Journal of New Approaches in Educational Research, 7(1), pp. 31-39. doi: 10.7821/naer.2018.1.248

Ethic (2017). ethic.es. Recuperado de: Diez claves para llevar la economía circular a la industria textil: https://ethic.es/2017/05/10-claves-llevar-la-economia-circular-la-industria-textil/

García-Mundo, L., Vargas-Enríquez, J., Genero, M., \& Piattini, M. (2015). Análisis de la evidencia existente sobre la influencia del uso de juegos serios en el aprendizaje en el área de la informática. ReVisión. Revista de investigación en Docencia de la Informática, 8(1). Recuperado de https://www.aenui.net/ojs/index.php?journal=revision\&page $=$ article\&op $=$ view\&path $\% 5 \mathrm{~B} \% 5$ $\mathrm{D}=172 \&$ path $\% 5 \mathrm{~B} \% 5 \mathrm{D}=295$

Ke, F. (2014). An implementation of design-based learning through creating educational computer games: A case study on mathematics learning during design and computing. Computers \& Education, 73, pp. 26-39. doi: 10.1016/j.compedu.2013.12.010

Marín-Díaz, V. (2015). La gamificación educativa. Una alternativa para la enseñanza creativa. Digital Education Review, 27. doi: 10.1344/der.2015.27

Naciones Unidas (2015). Transformar nuestro mundo: la Agenda 2030 para el desarrollo sostenible, resolución adoptada el 25 de septiembre de 2015 por la Asamblea General de las Naciones Unidas. AGNU $\mathrm{A} / \mathrm{RES} / 70 / 1$

Oliva, H. (2017). La gamificación como estrategia metodológica en el contexto educativo universitario. Realidad y Reflexión, 44, pp. 29-47. doi: 10.5377/ryr.v44i0.3563

Pisabarro, A., \& Vivaracho, C. (2018). Gamificación en el aula: yincana de programación. ReVisión. Revista de Investigación en Docencia de la Informática, 11(1), 3-4.

Villalustre, L., \& Del Moral, M. (2015). Gamificación: Estrategia para optimizar el proceso de aprendizaje y la adquisición de competencias en contextos universitarios. Digital Education Review, 27, pp. 13-31. doi: 10.1344/der.2015.27.13-31

Werbach, K., \& Hunter, D. (2015). The gamification toolkit: Dynamics, mechanics and components for the win. Philadelphia: Warton Digital Press. 